\title{
Hybrid FDG optimization method and kriging interpolator to optimize well locations
}

\author{
Gholamreza Khademi $^{1} \cdot$ Paknoosh Karimaghaee $^{1}$
}

Received: 17 May 2014/ Accepted: 17 May 2015/Published online: 30 May 2015

(C) The Author(s) 2015. This article is published with open access at Springerlink.com

\begin{abstract}
As the number of new significant oilfields discoveries are reduced and as production operations become more challenging and expensive, the efficient development of oil reservoirs in order to satisfy increasing worldwide demand for oil and gas becomes crucial. A key decision engineers must make is where to drill wells in the reservoir to maximize net present value or some other objectives. Since the number of possible solutions that depend on the size of reservoir can be very large, the use of an optimization algorithm is necessary. Optimization methods are divided into two main categories: non-gradient-based and gradient-based algorithms. In the former, the search strategy is to find global optimum while they need a great number of reservoir simulation runs. On the other hand, gradient-based optimization algorithms search locally but require fewer reservoir simulations. The computational cost of optimization method in the optimal well placement problem is substantial. Thus, in practical problems with large models, implying the gradient-based method is preferable. In the present paper, finite difference gradient (FDG) algorithm as one of the easy implemented gradientbased family is used. The main disadvantage of the mentioned technique is its dependency on the number of decision variables. The major contribution of this paper is to hybrid the FDG method and kriging interpolator. This interpolator is used as a proxy to decrease the required number of function evaluations and estimate the direction of movements in the FDG algorithm. Moreover, the idea of local grid refinement is proposed to eliminate the mixed
\end{abstract}

Gholamreza Khademi

khademi.gh@gmail.com

1 School of Electrical and Computer Engineering, Shiraz University, Shiraz, Iran integer problem of well placement. Then, the method is applied to some sample reservoirs and the simulation results verify the performance of the proposed method.

Keywords Optimal well placement · FDG optimization method $\cdot$ Kriging interpolator $\cdot$ Reservoir simulation

\section{Introduction}

In the context of oilfield development, production optimization has been an attractive research area in recent years. The motivation of such a considerable focus of attention is the need for producing limited existing fields as efficiently as possible, while decreasing economical and operating costs at the same time. Production optimization is often divided into well placement and well control optimization problems. In the well placement problems, the purpose is to drill wells at optimal locations so that more oil and gas can be extracted, while in well control problems, the well parameters such as producer or injector flow rates or bottom hole pressures (BHPs) are optimized.

The focus of this paper is on the literature review of optimal well placement procedure. Well placement is a challenging problem due to the existence of different decision variables e.g., well types and the presence of geological uncertainty which leads to multiple realization of reservoir. Thus, different possible solutions and scenarios exist, and only trusting to experienced reservoir engineers may lead to insufficient solution far from the optimal one. Consequently, the need for a systematic optimization method is obvious.

The literature relevant to optimal well placement is extensive. Numerous optimization methods to find optimal well place have been introduced over the past few years. 
These methods fall into two wide categories: gradient-free and gradient-based algorithms. Gradient-free methods are also categorized into stochastic algorithms, global search, and deterministic algorithms, local search strategy.

Both stochastic and deterministic algorithms do not need the derivatives of objective function respect to the decision variables. However, the stochastic approach requires a large number of objective function evaluations. Simulated annealing (Beckner and Song 1995), genetic algorithm (Yeten et al. 2003), particle swarm optimization (Onwunalu and Durlofsky 2010), the covariance matrix adaptation evolution strategy (CMAES) (Ding 2008) are popular stochastic optimization algorithms applied to the well placement problem. In Onwunalu (2010), standard PSO method is applied as an alternative to GA for well placement and showed that PSO resulted in better performance than GA. In addition, GPS (Isebor 2009), HJDS (Hooke and Jeeves 1961), polytope (Guyaguler 2002), and MADS (Ciaurri et al. 2011) are examples of deterministic local search methods. For detailed information on the implementation of the mentioned methods refer to (Mathworks 2009). Briefly, it can be concluded from the literature that stochastic methods are well suited for the well placement problem since normally the problem type is discrete. However, the disadvantage of this kind of optimization strategy covers its benefit such as global search optimum. The defect is the need for many forward reservoir simulation runs and also disability to improve objective function monotonically.

On the other hand, in gradient-based methods, the gradient of objective function subject to optimization variables is needed. This kind of optimization method is computationally efficient because it requires fewer function evaluations though it is capable of getting stuck in a local optimum. Gradient-based optimization methods are divided into two main groups in terms of calculating gradients. They include approximation and adjoint-based gradient algorithms. Well-known examples of gradient approximation method are simultaneous perturbation stochastic approximation (SPSA) and FDG. In Bangerth et al. (2006), the performance of FDG and SPSA methods is compared to the very fast simulated annealing (VFSA). They concluded that both FDG and SPSA algorithms are more efficient than gradient-free VSFA method. The main drawback of FDG and SPSA methods is that the step size along the search direction has to be chosen such that each function evaluation point corresponds to the lattice points in the simulation grid. Thus, a treatment to resolve the problem is to change the discrete optimization problem into continuous one. As a result, in Sarma and Chen (2008), adjoint method is suggested where the derivative of objective function is computed using the concept of optimal control theory and production optimization. In
Zhang et al. (2010), indirect optimal well placement based on the use of adjoint model and optimal well control is applied.

The complexity of adjoint-based algorithms in optimal well placement is similar to solving reservoir dynamic equations, which is the major drawback of the method. Since the problem is too complicated to compute gradients analytically, the simplest approach is to approximate gradients numerically using FDG or SPSA methods. In fact, it is easy to implement the method and the reservoir model is considered as a black box. In the present paper, FDG optimization method is used for optimal well placement. In the mentioned method, the gradients are computed using two-sided finite difference approximations for each optimization variables. Although the comparable accuracy of gradient calculations of FDG method over SPSA is shown in Bangerth et al. (2006), the performance of the underlying FDG optimization method may degrade for the large number of decision variables.

The key point of the present paper is to propose a hybrid optimization algorithm to eliminate drawbacks of FDG method. By introducing a linear interpolator called kriging and combine it to the FDG method, it is possible to approximate the objective function in order to find the gradients and estimate the direction of movement. This approach leads to significant decrease in reservoir simulations as the number of control variables increasing. Another problem of FDG method is the need for integer step size along search direction which is interpreted as a limitation. Thus, the idea of local gird refinement (LGR) is suggested. Eventually, in this paper, a gradient-based algorithm with few objective evaluations and free form the bound of just selecting the well locations in integer positions is achieved.

The paper is outlined as follows. In the "Well placement optimization methods" section, FDG optimization method and kriging interpolator are introduced. Net Present Value or NPV as the widely used objective function is described in "The objective function" section. In the "Hybrid FDG method and kriging interpolator" section, hybridizing the FDG algorithm and kriging interpolator is explained in details. "Simulation results" section is devoted to the simulation results. Finally, conclusions are discussed in the "Conclusion" section.

\section{Well placement optimization methods}

In the following "FDG optimization algorithm" subsection, FDG optimization algorithm for optimal well placement is explained. Then, in the "Kriging interpolator" subsection, mathematics of kriging interpolator has been presented. 


\section{FDG optimization algorithm}

The FDG algorithm can be considered as another definition of the steepest descent method, where the gradient vector is calculated using point-wise finite difference approximations in each of the directions associated with the decision variables. FDG optimization algorithm for a maximization problem is defined as follows:

1. Choose initial locations, $p_{0}$, for the wells and compute objective function $f\left(p_{0}\right)$.

2. Set $k=0, \gamma=0.101, \alpha=0.602$.

3. Compute $c_{k+1}=\left\lceil\frac{c}{(k+1)^{\gamma}}\right\rceil, a_{k+1}=\frac{\alpha}{(k+1)^{\alpha}}$.

4. Set evaluation points $s_{i}^{+}=p_{k}+c_{k+1} e_{i}$ with $e_{i}$ the unit vector in direction $i=1,2, \ldots, N$.

5. Evaluate $f_{i}^{+}=f\left(s_{i}^{+}\right)$.

6. Calculate the gradient approximation $g$ by $g_{i}=$ $\left(f_{i}^{+}-f\left(p_{k}\right)\right) / c_{k+1} e_{i}$.

7. Set $p_{k+1}=p_{k}+a_{k} g$.

8. Set $k=k+1$.

9. Stop condition is $k>K_{\max }$ or $\left\|p_{k+1}-p_{k}\right\|<\epsilon$, otherwise start from step 3 .

In the above algorithm, well positions out of the reservoir have been restricted. The exponent $\gamma$ and $\alpha$ control the speed convergence of the algorithm and their values are taken from Spall (2000). $N$ is the number of decision variables. The presented FDG method with a slight change from the Integer FDG (IFDG) defined in Bangerth et al. (2006) only needs $N+1$ function evaluations instead of $2 N$ times at each iteration. As the number of decision variables increase, more objective evaluations are required and this is the main problem of this method, which makes it insufficient for optimal placement of multiple wells.

It is notable that the gradient vector causes to have floating numbers for $p_{k+1}$ at step 7. Since well placement is a mixed integer problem, only the integer locations are acceptable. To solve the algorithm problem, one approach is that to round the gradient vector and the algorithm is called IFDG; however, this action may lead to inaccurate well positions and also cause to stop the algorithm in consecutive iterations. The remedy to this problem is to use the ability of Local Grid Refinement (LGR). With the aid of LGR, it is possible to divide each desired reservoir grid block into the smaller pieces to take into account the floating number and then drill the well more accurately in the optimum place. It is important to note that dividing the whole reservoir in smaller grids in order to have more accuracy in drilling will result in great computational burden since the numbers of grid blocks are significantly larger than the case of using LGR technique. In Fig. 1, implementation procedure of LGR is shown.

\section{Kriging interpolator}

Kriging is an interpolation method that is based on the computation of correlation among data points and is mainly used to estimate the unvisited points in the optimization search space. The most important advantages of Kriging are (1) providing standard errors of estimation for spatial data and (2) Identification of anisotropy parameters (directions of anisotropy and anisotropy ratio). In addition to that powerful tools, the kriging is a common method of interpolating unevenly sampled data. Actually, the kriging method can be assumed as a proxy which is incorporated into optimization procedures in order to decrease computational demands by reducing the required number of simulations. In other words, the interpolator helps FDG algorithm by estimating next direction of movements with fewer reservoir simulation runs. The algorithm in geostatistical applications is eliminated to three dimensions problem, however the method can be extended to n-dimensions.

Suppose that it is desired to estimate the quantity $Z$ and values $Z_{i}$ at $n$ points $(i=1,2, \ldots, n)$ with coordinates $\left(x_{i}, y_{i}\right)$ are measured. The purpose is to interpolate value $Z_{0}$ at point $\left(x_{0}, y_{0}\right)$. A general linear estimate can be written as

$Z^{*}\left(\mathrm{x}_{0}\right)=\sum_{i=1}^{n} \lambda_{i} Z\left(\mathrm{x}_{i}\right)$,

where the asterisk in $Z_{0}^{*}$ is used to differentiate the interpolated from the true value $Z_{0}$ and $\lambda_{i}$ are the weights.

Kriging provides an objective way of determining the weights $\lambda_{i}$ based on an understanding of the statistical properties of the data. To introduce kriging, one needs to get familiar with the spatial characterization of data. This is discussed in detail in Chiles and Delfinger (1999).

To characterize the spatial variations in data, it is useful to define a residual value that can be written as follows:

$Y\left(\mathrm{x}_{i}\right)=Z\left(\mathrm{x}_{i}\right)-m\left(\mathrm{x}_{i}\right)$,

where $m\left(\mathrm{x}_{i}\right)$ is a model of the large scale spatial trends in the data which might in the simplest case be just the population mean $\mu$.

Using the residual values, we can write a linear weighted interpolation of Eq. (1) as

$Z^{*}\left(\mathrm{x}_{0}\right)=Y^{*}\left(\mathrm{x}_{0}\right)+m\left(\mathrm{x}_{0}\right)$

$Y^{*}\left(\mathrm{x}_{0}\right)=\sum_{i=1}^{n} \lambda_{i} Y\left(\mathrm{x}_{i}\right)$

The main objective of kriging is to find the values of $\lambda_{i}$ that minimize the expected error variance of the estimate $Y^{*}$ which can be written as 

method
Fig. 1 Implementation of LGR

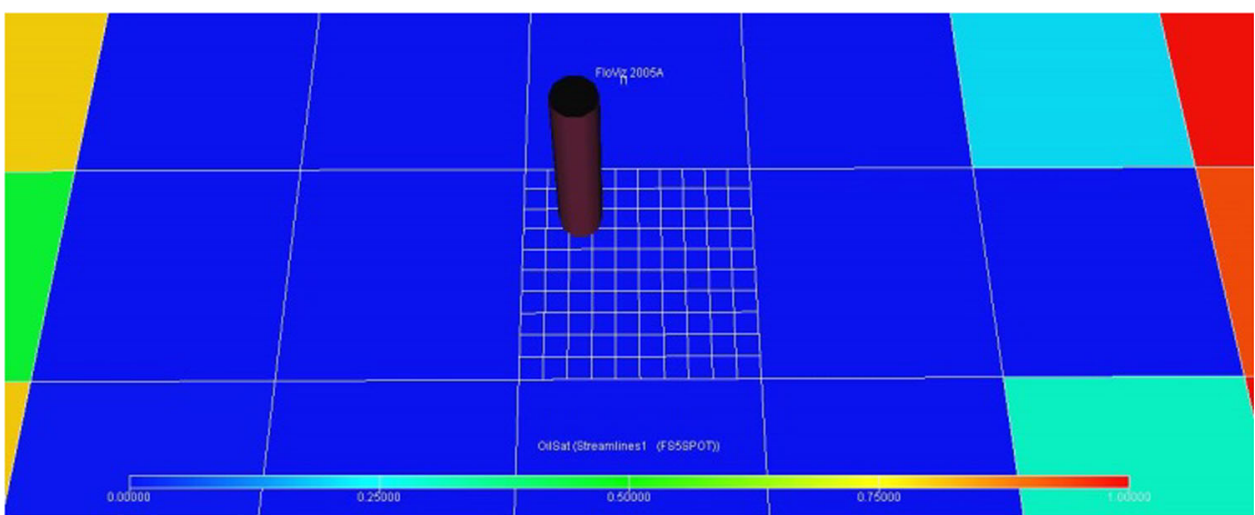

$e^{2}=E\left\{\left[Y^{*}\left(\mathrm{x}_{0}\right)-Y\left(\mathrm{x}_{0}\right)\right]^{2}\right\}$.

Different forms of kriging interpolator exist. Simple, ordinary, and universal kriging are various types of the mentioned method. In this paper, ordinary kriging is used since ordinary kriging is an accurate interpolator. In fact, at the point of data, the estimated value and the data value are equivalent. In the ordinary kriging, it is assumed that $m\left(\mathrm{x}_{i}\right)$ is an unknown mean. Then, besides the Eq. (11), an extra constraint which is described by (12) will be added to the problem.

$\sum_{i=1}^{n} \lambda_{i}=1$

According to the definition of spatial covariance and substitute it for the expected values, Eq. (8) is obtained.

$e^{2}=\sum_{i=1}^{n} \sum_{j=1}^{n} \lambda_{i} \lambda_{j} C\left(\mathrm{x}_{i}-\mathrm{x}_{j}\right)-2 \sum_{i=1}^{n} \lambda_{i} C\left(\mathrm{x}_{i}-\mathrm{x}_{0}\right)+C(0)$,

where $C(h)$ is spatial covariance and can be written as follows:

$C(h)=\frac{1}{m} \sum_{i=1}^{m}\left[Y\left(\mathrm{x}_{i}+h\right)\right]\left[Y\left(\mathrm{x}_{i}\right)\right]$.

If we have priori knowledge about the data points, it will be possible to calculate the spatial covariance. Thus, the only unknown parameters in Eq. (8) are $\lambda_{i}$. The weights can be found by solving a minimization problem i.e., minimize the error variance subject to $\lambda_{i}$. To do so, the derivative of Eq. (8) with respect to the weights is obtained and set it to zero.

$\frac{\partial e^{2}}{\partial \lambda_{i}}=2 \sum_{j=1}^{n} \lambda_{j} C\left(\mathrm{x}_{i}-\mathrm{x}_{j}\right)-2 C\left(\mathrm{x}_{i}-\mathrm{x}_{0}\right)=0$.

Finally the following equation is reached.

$\sum_{j=1}^{n} \lambda_{j} C\left(\mathrm{x}_{i}-\mathrm{x}_{j}\right)=C\left(\mathrm{x}_{i}-\mathrm{x}_{0}\right), \quad i=1,2, \ldots, n$.
This is done with the help of Lagrange multipliers. Moreover, the method implicitly evaluates the mean $\mu$. Briefly, the ordinary kriging system can be written as follows:

$$
\left\{\begin{array}{l}
\sum_{j=1}^{n} \lambda_{j} C\left(x_{i}-x_{j}\right)+\mu=C\left(x_{i}-x_{0}\right) \\
\sum_{j=1}^{n} \lambda_{j}=1
\end{array} \quad i=1,2, \ldots, n\right.
$$

The matrix form of Eq. (13) is described in (14).

$$
\begin{aligned}
& {\left[\begin{array}{cccc}
C\left(x_{1}-x_{1}\right) & \ldots & C\left(x_{1}-x_{n}\right) & 1 \\
\vdots & \ddots & \vdots & \vdots \\
C\left(x_{n}-x_{1}\right) & \cdots & C\left(x_{n}-x_{n}\right) & 1 \\
1 & \cdots & 1 & 0
\end{array}\right]\left[\begin{array}{c}
\lambda_{1} \\
\vdots \\
\lambda_{n} \\
\mu
\end{array}\right]} \\
& \quad=\left[\begin{array}{c}
C\left(x_{1}-x_{0}\right) \\
\vdots \\
C\left(x_{n}-x_{1}\right) \\
1
\end{array}\right] .
\end{aligned}
$$

In Eq. (14), the left-hand side matrix shows the dissimilarities among data points and the right vector in the Eq. (14) describes the dissimilarities between the estimation point and the data points. As you noticed, output of kriging interpolation method is always the estimated value of an unvisited point and estimation error variance. The estimation error variance can be used as a 
measure of how accurate is the kriging interpolation method. Estimation error variance can be obtained from Eq. (8).

In geostatistical applications, sampled data points are available and calculation of spatial variance based on the analysis of data is possible. While in this paper, there is no data from the first step of optimization algorithm, and at each iteration, the kriging dataset is updated whenever a new point is visited. Thus, the spatial variance cannot be computed. Consequently, a suitable power model is used for variance.

$C(h)=1-|h|^{1.5}$,

where $h$ is the distance between two points in twodimensional search space.

$h=\sqrt{\left(x_{i}-x_{0}\right)^{2}+\left(y_{i}-y_{0}\right)^{2}}$.

\section{The objective function}

The popular objective function used in the well placement optimization problem is Net Present Value or in short form NPV. The unit of NPV is dollar and taking into account the benefits and costs of extracting oil, gas, and water. More specifically, NPV is defined as the total benefit of oil production minus the total costs of water injection and production over the simulation time. Equation (17) describes the NPV objective function.

$J=\sum_{k=1}^{N_{t}}\left\{\sum_{j=1}^{N_{\text {prod }}}\left(\frac{r_{o} q_{o, j}^{k}-r_{w} q_{w, j}^{k}}{(1+b)^{t^{k} \beta 65}}\right) \Delta t^{k}-\sum_{i=1}^{N_{\mathrm{inj}}}\left[\left(\frac{q_{\mathrm{inj}, i}^{k}}{q_{\mathrm{inj}, i}^{k}+\beta}\right) C_{\mathrm{inj}}\right]\right\}$.

$N_{t}$ is the number of reservoir simulation time steps, $N_{\text {prod }}$ and $N_{\text {inj }}$ indicate the number of production and injection wells. $r_{o}$ is the oil revenue per unit volume, $r_{w}$ denotes the water disposal cost per unit. The unit for both $r_{o}$ and $r_{w}$ is \$/STB. $q_{o, j}^{k}$ and $q_{w, j}^{k}$ represent the oil and water rates of the $j$ th producer over the $k$ th simulation time steps, respectively. $t^{k}$ denotes the total simulation time in days and $\Delta t^{k}$ represents the size of the $k$ th time steps in days. $b$ is an annual discount factor and $C_{\text {inj }}$ is the required cost for drilling an injection well. $\beta$ is an adjustment parameter. Finally, $q_{\mathrm{inj}, i}^{k}$ shows the rate of $i$ th injection well over the $k$ th time steps (Zhang et al. 2010). In this paper, the goal is to consider the optimal well placement problem with fixed water injection rate. As a result, the second summation over the injectors which describes the total costs of water injection is ignored in the optimization problem.

\section{Hybrid FDG method and kriging interpolator}

As discussed, FDG optimization method is a gradientbased algorithm. The gradient is computed using pointwise finite difference approximations and implementation of method is simple. Performance of FDG optimization method may degrade for the large number of decision variables. In other words, in a problem with $N$ decision variables, $N+1$ simulation run is needed in each iteration which is not desirable. In this section, kriging interpolator as a proxy will be added to FDG method to reduce simulation runs. In fact, the kriging interpolator estimates $f_{i}^{+}=f\left(s_{i}^{+}\right)$in FDG method, so that it is possible to find the next direction of movement of the FDG algorithm. Moreover, estimation error variance is used to make sure interpolation is accurate enough. Prediction error is defined as estimation error standard deviation. Error standard deviation is the square of estimation error variance obtained from Eq. (8). If prediction error tends to zero, then estimation accuracy is $100 \%$. Prediction error is used as a criterion to determine whether the interpolated value is acceptable. Prediction error represents how much the estimated value is deviated from the actual value. In other words, Kriging standard deviation produces confidence interval for estimated values. At each iteration, $f\left(s_{i}^{+}\right)$is estimated by kriging interpolator and error variance of the estimate is obtained. If the prediction error was acceptable, the estimated value of $f\left(s_{i}^{+}\right)$would be trusting and is used to determine gradient. However, if the prediction error was more than a predefined value, the estimate will be discarded and $f\left(s_{i}^{+}\right)$will be obtained by simulation of the reservoir. An unvisited point with a large prediction error means that kriging interpolation does not have sufficient data points in the neighborhood of that point. Evaluation of that point by reservoir simulation and updating interpolation dataset helps kriging to be more precise in the next estimation. In overall, kriging interpolator decreases the number of objective function evaluation. Thus, the proposed hybrid gradient-based algorithm converges to local optimum very fast with minimum computational demand. Steps of hybridizing FDG and kriging are as follows:

1. Create an initial dataset by reservoir simulations and use them as the kriging interpolator dataset (Training Procedure).

2. Choose initial location.

3. Start FDG algorithm.

4. Evaluate $f\left(p_{k}\right)$ and then update kriging dataset.

5. Approximate $f\left(s_{i}^{+}\right)$values using kriging interpolator.

6. Calculate prediction error. 
- If prediction error is acceptable, use result of kriging interpolator.

- If prediction error is not acceptable, evaluate and update kriging dataset.

7. Generate next improved well location based on FDG algorithm.

8. Stop in case of convergence, otherwise, repeat from step 3.

It is crucial to note that the number of data points in the initial dataset depends on the size of search space. In other words, for a large reservoir, more initial data points are required to have an accurate approximation. Simulation results prove that the performance of hybrid FDG is definitely better than FDG in case of large number of decision variables. In Fig. 2, general flowchart of the proposed hybrid method is shown.

\section{Simulation results}

To consider a well placement optimization problem, Eclipse and Matlab software are used. In this section, the way of linking these software is explained. Then, rock and fluid properties of two simple reservoirs are given. Finally, the hybrid FDG algorithm is applied to illustrate the performance of the proposed method (FrontSim 2006).

\section{Link Matlab and Eclipse software}

Well placement optimization problem consists of two main parts: Optimization algorithm, Reservoir modeling, and simulation. Since reservoir model is complicated, it is conventional to use a suitable reservoir simulator. In this paper, FrontSim, one of the important modules of Eclipse software is used. In the FrontSim, the reservoir is modeled using streamline-based technique. In addition, in the

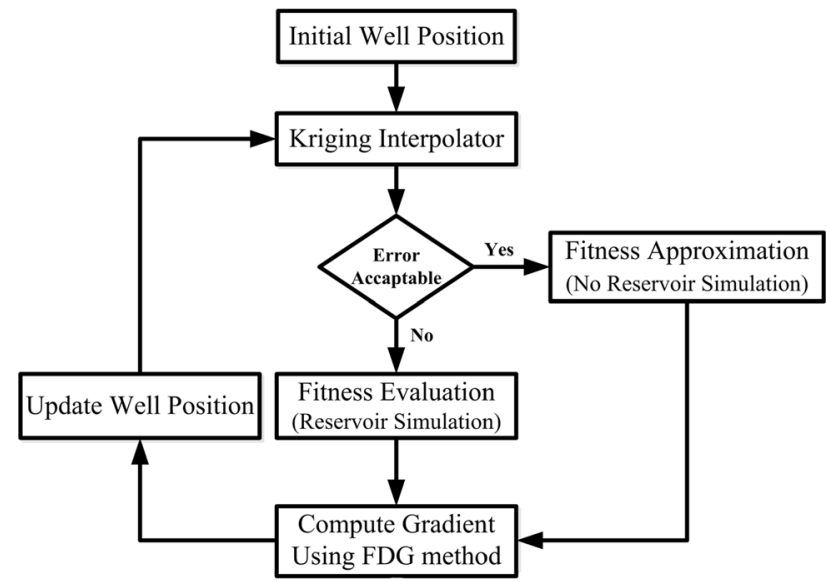

Fig. 2 Flowchart of hybrid FDG algorithm
Matlab software, the optimization algorithm is implemented. Figure 3 illustrates the flowchart of linking the Eclipse and Matlab software.

\section{Description of reservoir model and simulation}

Two different reservoirs have been chosen to verify the performance of the algorithm discussed in this paper. The first one is a simple five-spot reservoir and the second one is a complicated non-homogenous reservoir. The first scenario is to optimize locations of injection wells at the first reservoir, and the objective of next scenario is placement of producer wells in the second reservoir.

\section{Scenario 1: (injector placement)}

A homogenous oil-water reservoir of $761.9 \times 761.9 \times 10$ meters is considered. The reservoir is discretized by $32 \times$ $32 \times 1$ grid blocks of $23.81 \times 23.81 \times 10$ meters. The porosity distribution is homogenous and equals to 0.2 . Rock and fluid properties are given in Table 1 .

In the first scenario, there exist 5 vertical wells in the reservoir. One of them is injector and the rest are producers. The four producers are fixed in each of the corner

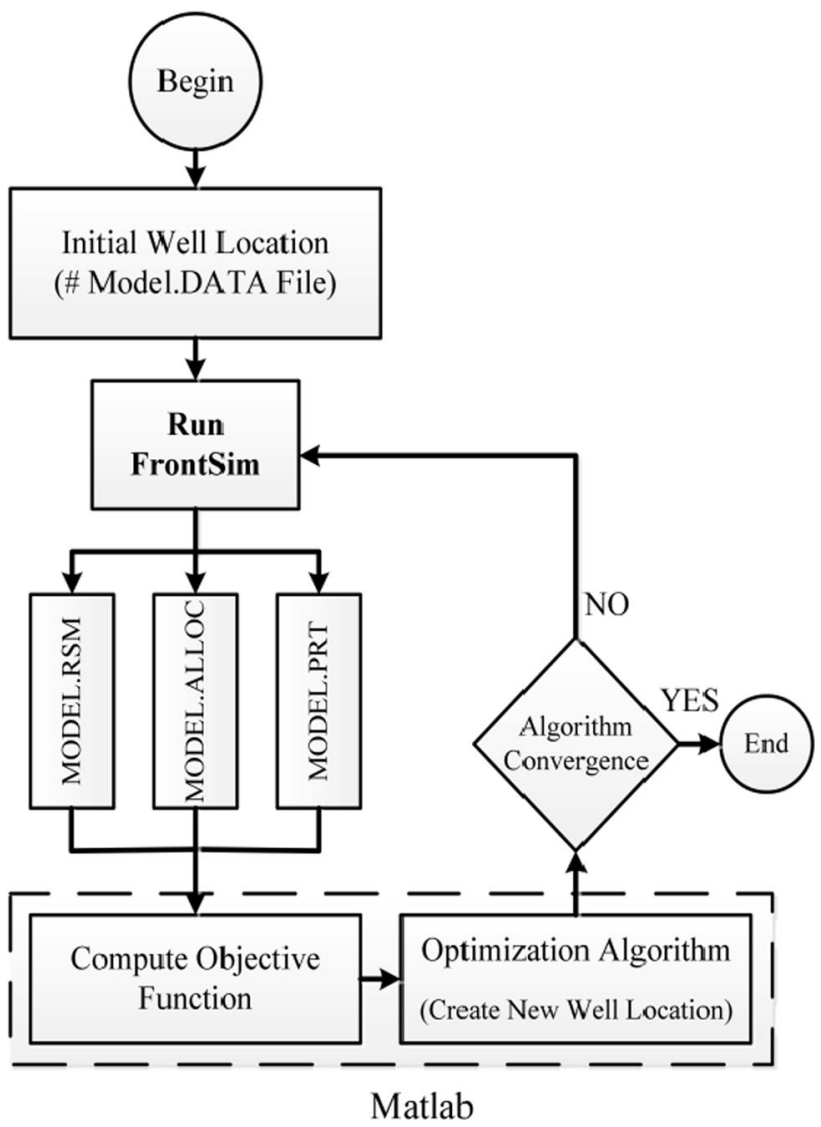

Fig. 3 Flowchart of linking Eclipse and Matlab 
Table 1 Rock and fluid properties of reservoir

\begin{tabular}{lll}
\hline Symbol & Value & Unit \\
\hline Grid cell dimensions & $32 \times 32 \times 1$ & $\mathrm{~m}^{3}$ \\
Initial pressure $\left(P_{i}\right)$ & 250 & $\mathrm{bar}$ \\
Water density $\left(\rho_{w}\right)$ & 1013.9 & $\mathrm{Kg} / \mathrm{m}^{3}$ \\
Oil density $\left(\rho_{o}\right)$ & 834.7 & $\mathrm{Kg} / \mathrm{m}^{3}$ \\
Gas density $\left(\rho_{g}\right)$ & 0.878 & $\mathrm{Kg} / \mathrm{m}^{3}$ \\
Water compressibility $\left(c_{w}\right)$ & $4.875 \mathrm{e}-05$ & $1 / \mathrm{bar}$ \\
Rock compressibility & $0.4 \mathrm{e}-05$ & $1 / \mathrm{bar}$ \\
Water viscosity $\left(\mu_{w}\right)$ & 0.5 & $\mathrm{cP}$ \\
Oil viscosity $\left(\mu_{o}\right)$ & 1.24 & $\mathrm{cP}$ \\
$B_{w}$ at atm pressure & 1.01 & $\mathrm{rm} / \mathrm{sm}^{3}$ \\
$B_{o}$ at atm pressure & 1.3 & $\mathrm{rm}^{3} / \mathrm{sm}^{3}$ \\
\hline
\end{tabular}

gridblocks. The injector rate target is $200 \mathrm{sm}^{3} /$ day and all the producers are controlled by reservoir volume rate target. The rate for producers is $75 \mathrm{sm}^{3} /$ day. The total simulation time is 3120 days.

The objective is to optimize the injector locations in the reservoir. The initial dataset used for kriging interpolator is 30 data points. A minimum predefined allowable error is specified. Whenever the estimation error exceeds the predefined value, objective function is evaluated and the kriging dataset is updated. FDG and hybrid FDG optimization algorithms are applied to the problem and the results in terms of number of required simulation runs are compared to each other. The found optimum location of the injector for both methods is close to each other and in the center of reservoir. The results are available in Table 2.

Two methods reach to the approximately the same optimum position for the same initial place where the global optimum NPV is $1.2824 \times 10^{5} \$$. In Fig. 4 , the value of FOPT per iteration in the hybrid FDG optimization is depicted. It is obvious that the algorithm improves the objective function. For better representation, only the 3 first steps and the optimum are shown. In FDG algorithm $N$ is 2 . Thus, the number of objective evaluation per iteration is 3 and the total simulations are $3 \times 9$, while in the hybrid FDG only 10 times the reservoir is simulated. However, it must be noted that 30 extra simulations for creating dataset in hybrid FDG method have been done. By taking it into account, the hybrid FDG needs $10+30$ evaluations which are more than the result of FDG. The differences of the proposed approach and simple FDG will be more visible when the number of decision variables increase. Thus, optimum placement of two injection wells is investigated.

This step is the same with the previous one except in the objective of optimization problem. In this case, the purpose is to optimize two injection wells with the rate target of 100 $\mathrm{sm}^{3} /$ day for each injector.
Table 2 Comparison of FDG with hybrid FDG

\begin{tabular}{lll}
\hline Algorithms & FDG & FDG + Kriging \\
\hline No. of simulations & 27 & 10 \\
Optimum NPV $\left(\times 10^{5} \$\right)$ & 1.2823 & 1.2821 \\
\hline
\end{tabular}

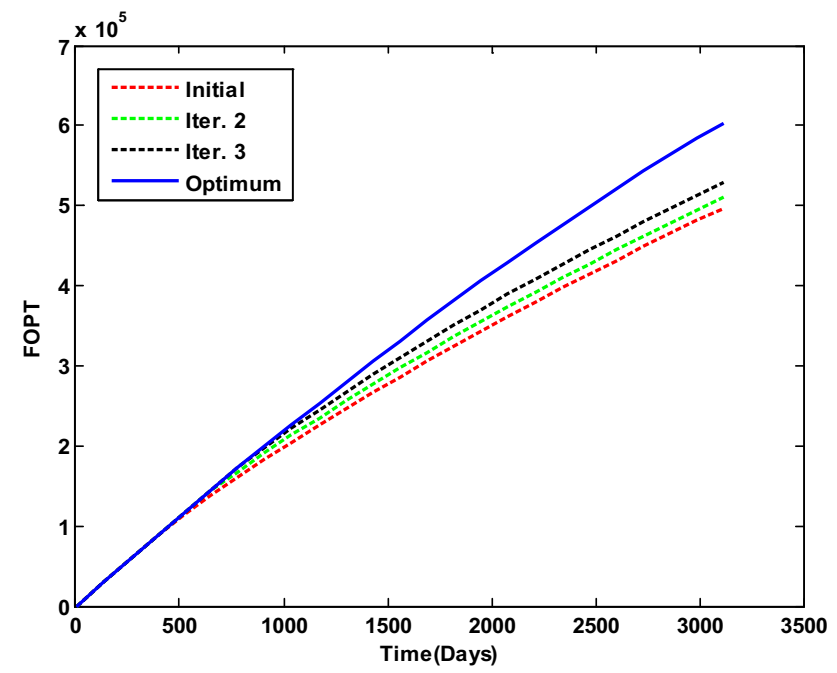

Fig. 4 Value of FOPT for one injector placement

Table 3 Comparison of FDG with hybrid FDG

\begin{tabular}{lll}
\hline Algorithms & FDG & FDG + Kriging \\
\hline No. of simulations & 175 & 43 \\
\hline
\end{tabular}

Two methods reach to the optimum locations which are placed approximately at the center of reservoir symmetrically. In FDG algorithm $N$ is 4 . Thus, the number of objective evaluation per iteration is 5 . The results are shown in Table 3.

The total numbers of FDG's simulation runs are 175 while this is only $43+30=73$ for the hybrid FDG with taking into account the number of initial simulation runs to create dataset for the interpolator. This scenario evidently shows better performance of the proposed method.

\section{Scenario 2: (producer placement)}

A non-homogenous oil-water-gas reservoir with dimension of $20 \times 40 \times 12$ grid blocks is considered. The gravity of the present phases, oil-water-gas, is 64.8-1.009760.91932 , respectively. The porosity distribution is also nonhomogenous. The rock compressibility at the reference pressure $\left(P_{\text {ref }}\right)$ of 3600 Psi is $10^{-6}\left(\frac{1}{\mathrm{Psi}}\right)$. Some of the main characteristics of this reservoir like equilibrium data are listed in the followings. 
Fig. 5 Ternary model of reservoir

Fig. 6 Oil saturation of reservoir
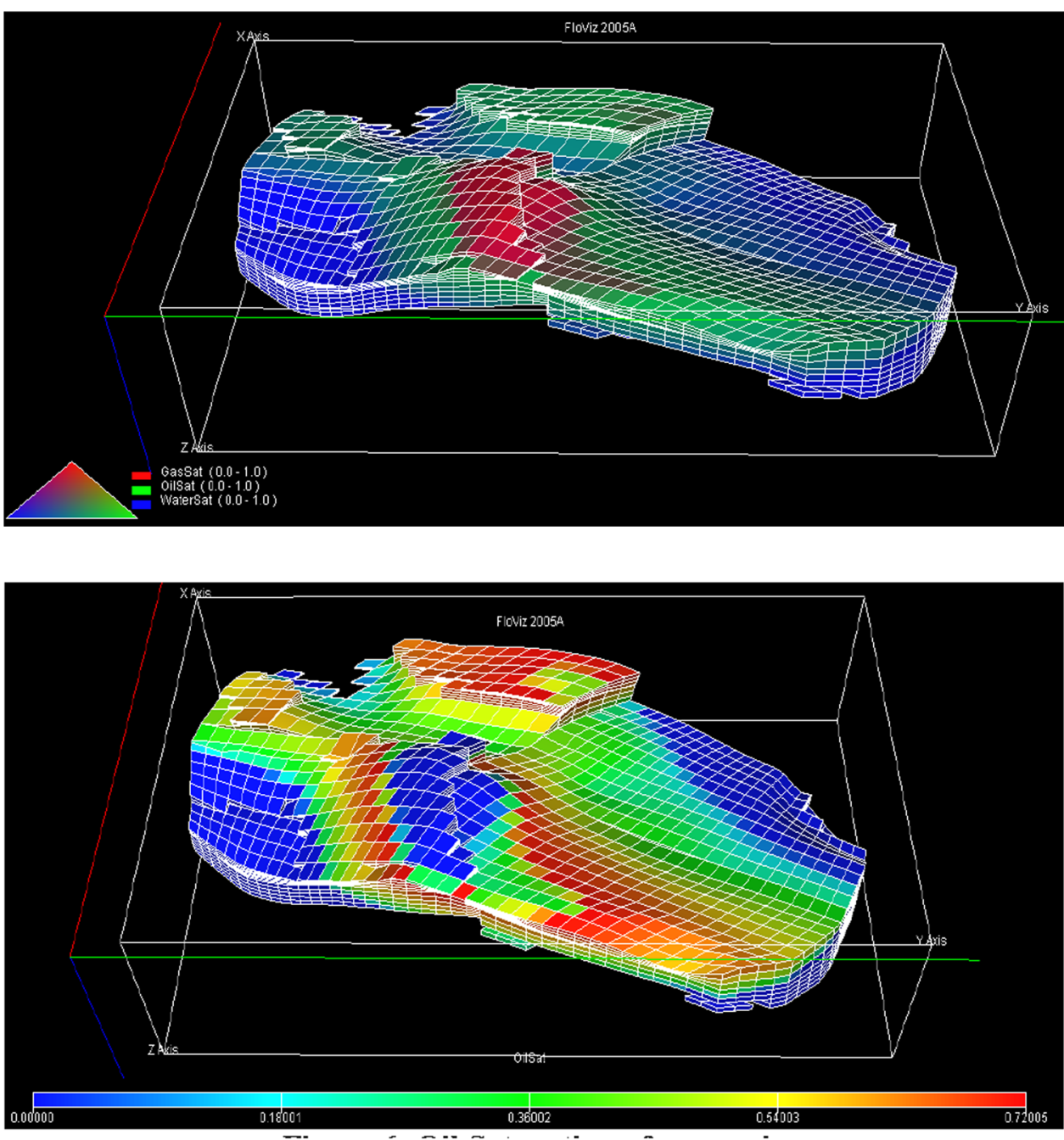

- Datum depth: $7920 \mathrm{ft}$.

- Pressure at datum depth: 4000 Psi.

- Depth of the water-oil contact: $8020 \mathrm{ft}$.

- Oil-water capillary pressure at the water-oil contact: 0 .

- Depth of the gas-oil contact: $7920 \mathrm{ft}$.

- Gas-oil capillary pressure at the gas-oil contact: 0 .

- Oil in place: $150 \times 10^{6} \mathrm{STB}$.

The model has a Fetkovich aquifer with datum depth of 8300 (ft) and initial volume of water of 10 billion STB. The aquifer productivity index is $1000 \mathrm{stb} /$ day/Psi. The ternary model and the oil saturation of reservoir are shown in Figs. 5 and 6, respectively.

In the second scenario, there are five horizontal producer wells in the field. The objective is that to optimize the location of a new vertical production well in order to increase field oil recovery efficiency (FOE). Each producer operates under bottomhole pressure control with the bottomhole pressures all fixed equal to 2000 psi. The total simulation time is 3 years. Before solving the optimization problem, an exhaustive run has been done by putting the

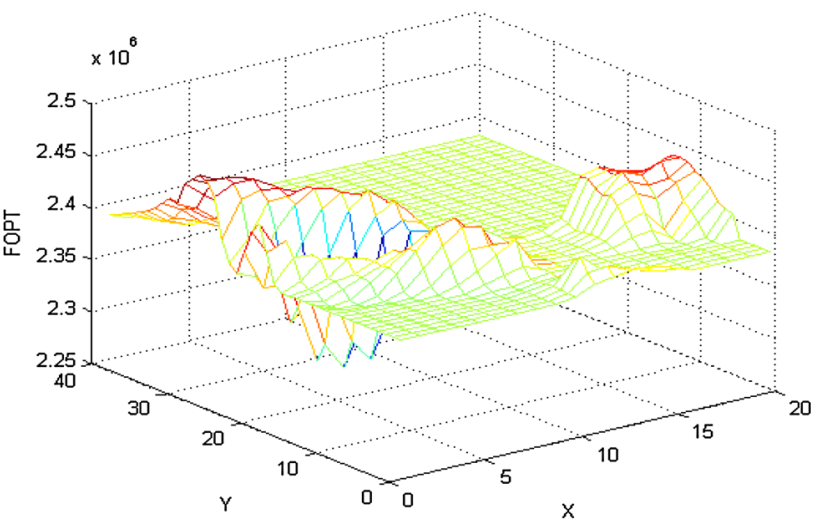

Fig. 7 Placement of a producer well by using an exhaustive run at scenario 2

new production well in each grid block and FOPT is calculated. The result of exhaustive run is shown in Fig. 7. It is obvious that there are multiple local optimum locations for the new production well. The main drawback of FDG method is that the algorithm may stick in local optimums. 
Table 4 Comparison of kriging with linear and cubic interpolation method

\begin{tabular}{|c|c|c|c|c|}
\hline Well position & FOPT $\left(m^{3}\right)$ & Kiriging (\% error) & Cubic (\% error) & Linear (\% error) \\
\hline$(15,15)$ & $2,389,208$ & $\underline{\mathbf{0 . 5 3 9 0}}$ & 0.8073 & 0.7869 \\
\hline$(3,38)$ & $2,380,258$ & 0.1635 & $\underline{\mathbf{0 . 1 5 8 5}}$ & 0.1819 \\
\hline$(5,32)$ & $2,382,608$ & $\underline{0.0086}$ & 0.1613 & 0.1522 \\
\hline$(15,14)$ & $2,384,796$ & $\underline{1.0548}$ & 1.1915 & 1.0599 \\
\hline$(11,17)$ & $2,380,584$ & 0.1416 & $\underline{0.1140}$ & 0.2044 \\
\hline$(6,22)$ & $2,438,362$ & -1.5221 & -1.6102 & -1.5621 \\
\hline$(18,37)$ & $2,380,243$ & $\underline{0.0187}$ & - & - \\
\hline$(9,40)$ & $2,380,255$ & $\underline{0.0078}$ & - & - \\
\hline$(20,7)$ & $2,404,542$ & -0.2982 & - & - \\
\hline$(20,16)$ & $2,416,680$ & $\underline{0.0857}$ & - & - \\
\hline
\end{tabular}

Although there are many well-known interpolation methods such as cubic, linear, nearest, and splines, kriging method is chosen for optimal well placement. As earlier discussed, kriging interpolation is the most appropriate method when you are dealing with irregular and unevenly sampled data. To verify the efficiency of kriging method, the method is compared to other common interpolation approaches such as linear and cubic method. Reservoir FOPT is interpolated for different unvisited positions of producer well. The initial dataset used for interpolation is 30 data points. Estimation errors of kriging, linear and cubic method are given in Table 4 . The method with smallest estimation error is highlighted and underlined. Moreover, the actual value of FOPT at each well position is given in column two.

It can be concluded that kriging interpolation method is more accurate in most cases. The main purpose of this comparison is to show that linear and cubic methods tend to overshoot with unevenly spaced data. Unsatisfactory result of linear and cubic interpolation method which is overshot is shown by dash. Then, Integer FDG and hybrid Integer FDG optimization algorithms are applied to the problem and the results are shown in Table 5.

Two methods reach to the approximately the same optimum position for the same initial place. In the next step, locations of two production wells are optimized to verify better performance of hybrid IFDG over IFDG in terms of reducing simulation runs. The result is provided in Table 6.

\section{Conclusion}

Well placement optimization problem is divided into two main categories: gradient-free and gradient-based optimization methods. The gradient-free algorithm needs no information about the objective function's derivative but
Table 5 Comparison of IFDG with hybrid IFDG

\begin{tabular}{lll}
\hline Algorithms & IFDG & IFDG + Kriging \\
\hline No. of simulations & 24 & 8 \\
FOE & 0.016170 & 0.016121 \\
\hline
\end{tabular}

Table 6 Comparison of FDG with hybrid FDG

\begin{tabular}{lll}
\hline Algorithms & IFDG & IFDG + Kriging \\
\hline No. of simulations & 55 & 14 \\
\hline
\end{tabular}

require many simulation runs to achieve the optimum location. While in the gradient-based method, the gradient and fewer simulation runs are needed. In this paper, FDG optimization method is chosen since implementation of this gradient-based method is simple and also accurate and totally require less evaluation than the stochastic optimization method. However, the main drawback of FDG is its dependency on the number of decision variables which makes it insufficient for placement of several wells.

In the present paper, a hybrid optimization method is used to decrease the required number of simulations. In the proposed hybrid method, FDG algorithm and kriging interpolator are combined. The role of kriging interpolator is to help FDG method to approximate the gradients. This action leads to fewer simulation runs. After introducing the proposed approach, the NPV objective function is defined. Finally, the hybrid optimization method is applied to a sample reservoir. Results of FDG and hybrid FDG are compared to each other for two scenarios and it has been concluded that both algorithms achieve to nearly the same optimum, while the number of simulation runs in the hybrid FDG algorithm is significantly fewer than the FDG method. 
Open Access This article is distributed under the terms of the Creative Commons Attribution 4.0 International License (http:// creativecommons.org/licenses/by/4.0/), which permits unrestricted use, distribution, and reproduction in any medium, provided you give appropriate credit to the original author(s) and the source, provide a link to the Creative Commons license, and indicate if changes were made.

\section{References}

Bangerth W, Klie H, Wheeler MF, Stoffa PL, Sen MK (2006) On optimization algorithms for the reservoir oil well placement problem. Comput Geosci 10:303-319

Beckner BL, Song X (1995) Field development planning using simulated annealing-optimal economic well scheduling and placement. Paper SPE 30650 presented at the SPE Annual Technical Conference and Exhibition held in Dallas, Texas, USA, 22-25 Oct 1995

Chiles JP, Delfinger P (1999) Geostatistics-modeling spatial uncertainty. Wiley, New York

Ciaurri DE, Isebor OJ, Durlofsky LJ (2011) Application of derivativefree methodologies to generally constrained oil production optimization problems. Int $\mathbf{J}$ Math Model Numer Optim 2:134-161

Ding DY (2008) Optimization of well placement using evolutionary algorithms. Paper SPE 113525 presented at the SPE Europec/ EAGE Annual Conference and Exhibition, Rome, Italy, 9-12 June 2008

FrontSim (2006) Refrence manual and technical description. Schlumberger GeoQuest, Houston
Guyaguler B (2002) Optimization of well placement and assessment of uncertainty. PhD thesis, Department of Petroleum Engineering, Stanford University

Hooke R, Jeeves TA (1961) Direct search solution of numerical and statistical. Journal of the ACM 8:212-229

Isebor OJ (2009) Constrained production optimization with an emphasis on derivative-free. Master's thesis, Department of Energy Resources Engineering, Stanford University

Mathworks (2009) Genetic algorithm and direct search toolbox. Users Guide

Onwunalu JE (2010) Optimization of field development using particle swarm optimization and new well pattern descriptions. PhD thesis, Department of Energy Resources Engineering, Stanford University

Onwunalu JE, Durlofsky LJ (2010) Application of a particle swarm optimization algorithm for determining optimum well location and type. Comput Geosci 14:183-198

Sarma P, Chen WH (2008) Efficient well placement optimization with gradient based algorithms and adjoint. Paper SPE 112257 presented at the SPE, Intelligent Energy Conference and Exhibition held in Amsterdam, The Netherlands, Feb 2008

Spall JC (2000) Adaptive stochastic approximation by the simultaneous perturbation method. IEEE Trans Autom Control 45:1839-1853

Yeten B, Durlofsky LJ, Aziz K (2003) Optimization of nonconventional well type, location and trajectory. SPE J 8:200-210

Zhang K, Li G, Reynolds AC, Yao J, Zhang L (2010) Optimal well placement using an adjoint gradient. J Pet Sci Eng 73:220 